\title{
Polytopes and the Existence of Approximate Equilibria in Discontinuous Games*
}

\author{
Guilherme Carmona ${ }^{\dagger}$ \\ Universidade Nova de Lisboa
}

May 26, 2009

\begin{abstract}
Radzik (1991) showed that, by strengthening the usual quasi-concavity assumption on players' payoff functions, upper semi-continuous two-player games on compact intervals of the real line have $\varepsilon$-equilibria for all $\varepsilon>0$. Ziad (1997) then stated that the same conclusion holds for $n$-player games on compact, convex subsets of $\mathbb{R}^{m}, m \geq 1$, provided that the upper semi-continuity condition is strengthened. Both Radzik's and Ziad's proofs rely crucially on the lower hemi-continuity of the $\varepsilon$-best reply correspondence. We show that: (1) in contrast to what is stated by Ziad, his conditions fail to be sufficient for the lower hemi-continuity of the approximate best-reply correspondence, (2) the approximate best-reply correspondence is indeed lower hemi-continuous if players' action spaces are polytopes, and (3) with action spaces as polytopes, Ziad's theorem can be stated so that it properly generalizes Radzik's theorem.
\end{abstract}

*I wish to thank Erik Balder, Paulo Bárcia, Paulo Côrte-Real, Branko Grünbaun, Gil Kalai, Armando Machado, Luís Vasconcelos and an anonymous referee for very helpful comments. Special thanks are due to an associate editor, whose comments greatly improved the paper. I thank also John Huffstot for editorial assistance. Any remaining error is, of course, my own.

${ }^{\dagger}$ Address: Universidade Nova de Lisboa, Faculdade de Economia, Campus de Campolide, 1099032 Lisboa, Portugal; Phone: (351) 21380 1672; Fax: (351) 21387 0933; email: gcarmona@fe.unl.pt. 
Journal of Economic Literature Classification Numbers: C72.

Keywords: Discontinuous games, upper semi-continuity, lower semi-continuity, quasi-concavity, approximate equilibrium.

\section{Introduction}

Classic existence results (e.g., Nash (1950)) show that a normal-form game has a Nash equilibrium provided that two sets of conditions hold. First, each player's action space is a non-empty, compact, convex subset of a euclidean space. Second, each player's payoff function is jointly continuous and separately quasi-concave in his own action. The goal of this note is to show that, for the existence of approximate equilibria in normal-form games with polytopes as action spaces, the continuity requirement on players' payoff functions can be weakened from joint continuity to joint upper semi-continuity by strengthening the quasi-concavity requirement.

Our results build upon those of Radzik (1991) and Ziad (1997). In order to explain our results and how they relate to theirs, we briefly recall some game-theoretical and mathematical notions (Definitions 1 and 2, respectively).

Definition 1 1. A normal form game $G$ consists of a finite set of players $N=$ $\{1, \ldots, n\}$, and, for all players $i \in N$, a pure strategy set $X_{i}$, which is a nonempty compact subset of $\mathbb{R}^{m}$, and a bounded payoff function $u_{i}: X \rightarrow \mathbb{R}$, where $X=\times_{i \in N} X_{i}$.

2. The symbol $-i$ denotes "all players but $i$." In particular, $X_{-i}=\times_{j \neq i} X_{j}$. The value function of player $i$ is $V_{i}: X_{-i} \rightarrow \mathbb{R}$ defined by $V_{i}\left(x_{-i}\right)=\sup _{x_{i} \in X_{i}} u_{i}\left(x_{i}, x_{-i}\right)$.

3. Given a game $G$ and $\varepsilon \geq 0$, an $\varepsilon$-equilibrium of $G$ is $x^{*} \in X$ such that $u_{i}\left(x^{*}\right) \geq$ $u_{i}\left(x_{i}, x_{-i}^{*}\right)-\varepsilon$ for all $i \in N$ and $x_{i} \in X_{i}$. A Nash equilibrium of $G$ is an $\varepsilon$-equilibrium for $\varepsilon=0$.

4. For all $\varepsilon>0$ and $i \in N$, the player $i$ 's $\varepsilon$-best-reply correspondence is $B R_{i}^{\varepsilon}$ : $X_{-i} \rightrightarrows X_{i}$ defined by $B R_{i}^{\varepsilon}\left(x_{-i}\right)=\mathrm{cl}\left(\left\{x_{i} \in X_{i}: u_{i}\left(x_{i}, x_{-i}\right)>\sup _{\hat{x}_{i} \in X_{i}} u_{i}\left(\hat{x}_{i}, x_{-i}\right)-\right.\right.$ 
$\varepsilon\})$ for all $x_{-i} \in X_{-i} \cdot{ }^{1}$ The $\varepsilon$-best-reply correspondence is $B R_{\varepsilon}: X \rightrightarrows X$ defined by $B R_{\varepsilon}(x)=B R_{1}^{\varepsilon}\left(x_{-1}\right) \times \cdots \times B R_{n}^{\varepsilon}\left(x_{-n}\right)$ for all $x \in X$.

Definition 2 Let $X$ and $Y$ be compact and convex subsets of a euclidean space.

1. A polytope is the convex hull of finitely many points.

2. For a function $f: X \rightarrow \mathbb{R}$, an upper set is of the form $\{x \in X: f(x) \geq a\}$ for some $a \in \mathbb{R}$.

3. A function is upper semi-continuous if all its upper sets are closed, quasiconcave if all its upper sets are convex, and polyhedral quasi-concave if all its upper sets are polytopes.

4. For a function $f: X \times Y \rightarrow \mathbb{R}$, these notions apply jointly to the argument $(x, y)$ of $f(x, y)$. Separate upper semi-continuity, quasi-concavity and polyhedral quasi-concavity in $x$, say, apply to $f(x, y)$ as a function of $x$.

5. A correspondence $\beta: X \rightrightarrows Y$ is closed-valued if $\beta(x)$ is closed for all $x \in X$. It is lower hemi-continuous if the set $\{x \in X: \beta(x) \subseteq F\}$ is closed for each closed subset $F$ of $Y$. A closed-valued correspondence is upper hemi-continuous if its graph is closed.

6. A point $x \in X$ is a fixed point of $\beta$ if $x \in \beta(x)$. A function $f: X \rightarrow Y$ is a continuous selection of $\beta$ if $f$ is continuous and $f(x) \in \beta(x)$ for all $x \in X$.

Recall, as well, that if $\beta: X \rightrightarrows X$ is such that $\beta(x)$ is nonempty, compact and convex for all $x \in X$ and $X$ if finite-dimensional and compact, then: (1) If $\beta$ is upper hemi-continuous, by Kakutani's fixed point theorem there is a fixed point $x$ of $\beta$. (2) If $\beta$ is lower hemi-continuous, then Michael's selection theorem implies that there is a continuous selection $f$ of $\beta$.

Radzik (1991) considers payoff functions that are jointly upper semi-continuous and separately quasi-concave in each player's own action. Radzik considers only two players, each with an interval as strategy set. He shows that these assumptions

\footnotetext{
${ }^{1}$ For all subsets $A$ of a topological space $Y, \operatorname{cl}(A)$ denotes the closure of $A$.
} 
are not enough to guarantee the existence of an $\varepsilon$-equilibrium for all $\varepsilon>0$. A solution to this existence problem can be obtained by strengthening upper semicontinuity to continuity since this would imply the existence of a Nash equilibrium using Kakutani's theorem. Radzik obtained an alternative solution by strengthening separate quasi-concavity to the following notion of piecewise quasi-concavity: For player 1 , the compact interval $X_{2}$ that is the strategy set of player 2 is suitably covered by a finite number of intervals $\left[b_{i}, b_{i+1}\right]$ so that player 1 's payoff function $u_{1}$ is jointly quasi-concave on $X_{1} \times\left[b_{i}, b_{i+1}\right]$ for all $i$ (piecewise quasi-concavity of player 2's payoff function is defined analogously). ${ }^{2}$ His main result shows that if each player's payoff function is jointly upper semi-continuous and piecewise quasi-concave, then the game has an $\varepsilon$-equilibrium for all $\varepsilon>0$.

Radzik proves his result by establishing that the $\varepsilon$-best reply correspondence is lower hemi-continuous, with nonempty, compact and convex values. Michael's selection theorem then implies that $\varepsilon$-best reply correspondence has a continuous selection, to which we can apply Brouwer's fixed point theorem to obtain an $\varepsilon$-equilibrium.

Ziad (1997) also considers the existence of approximate equilibrium in normalform games. His setting is more general than that of Radzik (1991) since it allows for $n$ players (not just two) and nonempty, convex, compact subsets of euclidean spaces as strategy spaces (not just compact intervals on the real line). He also extends the notion of piecewise quasi-concavity to this more general setting as follows: The finite cover of $X_{-i}$ by closed intervals is generalized to a finite cover by convex compact sets $X_{-i}^{l}$, so that $u_{i}$ is jointly quasi-concave on $X_{i} \times X_{-i}^{l}$. For ease of reference, this notion is presented in the following definition.

Definition 3 Player i's payoff function is piecewise quasi-concave if there is a finite cover of $X_{-i}$ by compact convex subsets $X_{-i}^{l}$ such that $u_{i}$ is quasi-concave on $X_{i} \times X_{-i}^{l}$ for all $l .{ }^{3}$

\footnotetext{
${ }^{2}$ Radzik has named this property strong quasi-concavity. While the property is a strengthening of separate quasi-concavity in each player's own action, the term strong quasi-concavity suggests that it is a strengthening of joint quasi-concavity, which it is not. For this reason, we refer to it as piecewise quasi-concavity.

${ }^{3}$ When $n=2$ and players' action spaces are intervals in $\mathbb{R}$, this definition collapses into Radzik's
} 
He then claims, as his main result, that the game has an $\varepsilon$-equilibrium for all $\varepsilon>0$ if each player's payoff function $u_{i}$ is upper semi-continuous, piecewise quasi-concave and if it satisfies an additional property called $i$-upper semi-continuity. Definition 4 presents its definition, which will be discussed below.

Definition 4 Player $i$ 's payoff function is $i$-upper semi-continuous if

$$
\limsup _{k} V_{i}\left(\alpha_{k} z_{-i}^{k}+\left(1-\alpha_{k}\right) x_{-i}\right) \leq \underset{k}{\limsup } V_{i}\left(z_{-i}^{k}\right)
$$

for all $x_{-i} \in X_{-i},\left\{z_{-i}^{k}\right\}_{k=1}^{\infty}$ converging to $x_{-i}$ and $\left\{\alpha_{k}\right\}_{k=1}^{\infty} \subseteq(0,1]$ converging to zero.

Our contributions to the problem of existence of approximate equilibrium in normal-form games are the following: First, to note the following mistake in Ziad's paper: while he claims that the $\varepsilon$-best reply correspondence is upper hemi-continuous, his argument in the proof of the theorem attempts, in effect, to show that such a correspondence is lower hemi-continuous. Consequently, Ziad's argument would need Michael's selection theorem rather than Kakutani's fixed point theorem (as was the case in Radzik (1991)).

Second, we show that, under Ziad's assumptions, the $\varepsilon$-best reply correspondence may however fail to be lower hemi-continuous and to have a continuous selection. This implies that Ziad's argument does not prove the existence result he claims.

Third, we show that in Ziad's framework the $\varepsilon$-best reply correspondence is indeed lower hemi-continuous if players' action spaces are polytopes. This implies that Ziad's existence theorem is valid for games with polytopes as action spaces.

Fourth, we show that this modified version of Ziad's result (with polytopes as action spaces) implies Radzik's result. Recall that those two results differ because Ziad's result requires an additional condition on players' payoff functions ( $i$-upper semi-continuity, Definition 4 above) while it allows for more than two players and for more general action spaces. We show that under Radzik's assumptions, players' payoff functions are $i$-upper semi-continuous, which immediately implies that Radzik's result follows from that of Ziad.

notion of piecewise quasi-concavity. 
Fifth, we show that $i$-upper semi-continuity is implied by the following requirement on players' value functions:

Definition 5 Player $i$ 's value function is piecewise polyhedral quasi-concave if there is a finite cover of $X_{-i}$ by compact convex subsets $X_{-i}^{l}$ such that $V_{i}$ is polyhedral quasiconcave on $X_{-i}^{l}$ for all $l$.

That is, we show that if player $i$ 's value function is piecewise polyhedral quasi-concave, then his value function is $i$-upper semi-continuous. ${ }^{4}$ Furthermore, we show that, in Radzik's setting each player's value function is piecewise polyhedral quasi-concave. Thus, the sufficiency of the piecewise polyhedral quasi-concavity of $V_{i}$ for the $i$-upper semi-continuity of $u_{i}$ allows us to state an existence result for approximate equilibria of $n$-person games that simultaneously dispenses with the lower semi-continuity of $u_{i}$ altogether and generalizes Radzik's theorem.

Proposition 6 Let $G$ be an n-player game such that for all $i \in N$, (1) $X_{i}$ is a polytope, (2) $u_{i}$ is upper semi-continuous and piecewise quasi-concave (Definitions 2 and 3), and (3) $V_{i}$ is piecewise polyhedral quasi-concave (Definition 5). Then, G has an $\varepsilon$-equilibrium for all $\varepsilon>0$.

In conclusion, in order to obtain the existence of an approximate equilibrium, the continuity of players' payoff function can be weakened to upper semi-continuity. This is the case when players' action spaces are polytopes and when players' payoff and value functions satisfy a form of quasi-concavity stronger than separate quasiconcavity in each player's own action. The usefulness of Proposition 6 therefore lies in games whose players' payoff functions do not satisfy any form of lower semi-continuity, but which are, in compensation, upper semi-continuous and satisfy a relatively strong

\footnotetext{
${ }^{4}$ Clearly, $i$-upper semi-continuity is implied by the continuity of $V_{i}$ and so can be thought of as a weak form of continuity. Note, however, that $i$-upper semi-continuity is neither implied nor does it imply upper semi-continuity. Indeed, player 2's payoff function in Example 11 below is upper semi-continuous but not $i$-upper semi-continuous. Conversely, if $X_{1}=X_{2}=[0,1]$ and $u_{1}$ is defined by $u_{1}\left(x_{1}, x_{2}\right)=1$ if $x_{1} \leq 1 / 2$ and $u_{1}\left(x_{1}, x_{2}\right)=2$ if $x_{1}>1 / 2$, then $u_{1}$ is $i$-upper semi-continuous but not upper semi-continuous.
} 
form of quasi-concavity. In contrast, if players' payoff functions are discontinuous and separately quasi-concave in each player's own action but satisfy no stronger quasiconcave property, then one has to rely on the results of Dasgupta and Maskin (1986) and Reny (1999) to establish the existence of equilibria.

Finally, we note that Proposition 6 is related to Theorem 2 of Gale, Klee, and Rockafellar (1968), which, in particular, states that, for all closed convex subsets $D$ of a finite-dimensional Euclidean space, every convex function on $D$ is upper semicontinuous if and only if the intersection of $D$ with any polytope is a polytope. This result implies that every concave function on a polytope is lower semi-continuous. ${ }^{5}$ This, in turn, implies the following existence result: If $G$ is an $n$-player game such that for all $i \in N,(1) X_{i}$ is a polytope, (2) $u_{i}$ is upper semi-continuous and (3) there exists a finite cover of $X_{-i}$ by polytopes $X_{-i}^{l}$ such that $u_{i}$ is concave on $X_{i} \times X_{-i}^{l}$ for all $l$, then $G$ has a Nash equilibrium. In fact, by their Theorem $2, u_{i}$ would be lower semi-continuous on $X_{i} \times X_{-i}^{l}$ for all $l$, and so on $X=X_{i} \times X_{-i}$. Together with the upper semi-continuity of $u_{i}$, this implies that $u_{i}$ is actually continuous and the existence of equilibrium follows from Kakutani's fixed point theorem. Thus, in comparison, Proposition 6 allows for compact convex partitions of $X_{-i}$ and it requires only quasi-concavity of $u_{i}$ on $X_{i} \times X_{-i}^{l}$ but it only guarantees the existence of approximate equilibrium and it requires $V_{i}$ to be polyhedral quasi-concave on $X_{-i}^{l}$. More importantly, Proposition 6 allows for discontinuous payoff functions whereas the above existence result applies only to payoff functions that are, in effect, continuous.

\footnotetext{
${ }^{5}$ Indeed, if $D$ is a polytope, then $D$ is convex and closed and its intersection with a polytope is also a polytope. Furthermore, if $f: D \rightarrow \mathbb{R}$ is concave, then $-f$ is convex. Thus, Theorem 2 of Gale, Klee, and Rockafellar (1968) implies that $-f$ is upper semi-continuous and so $f$ is lower semi-continuous.
} 


\section{Characterizations of Polytopes and Ziad's The- orem}

We start by stating that Ziad's theorem is valid when players' action spaces are polytopes.

Theorem 7 (Ziad) Let $G$ be an n-player game such that for all $i \in N$, (1) $X_{i}$ is a polytope and (2) $u_{i}$ is upper semi-continuous, $i$-upper semi-continuous and piecewise quasi-concave. Then for each $\varepsilon>0$, each player's $\varepsilon$-best reply correspondence is lower hemi-continuous. Consequently, $G$ has an $\varepsilon$-equilibrium for all $\varepsilon>0$.

The reason why polytopes are important for Ziad's theorem is given in Lemma 9, which is a simple consequence of the characterization of polytopes that we provide in Proposition 8. The importance of Lemma 9 is that it allows us to establish the lower hemi-continuity of the $\varepsilon$-best reply correspondence (which, as explained in the introduction, implies the existence of an $\varepsilon$-equilibrium together with Michael's selection theorem and Brouwer's fixed point theorem).

We start by providing two characterizations of polytopes.

Proposition 8 Let $P \subseteq \mathbb{R}^{n}$. Then, the following conditions are equivalent:

1. $P$ is a polytope;

2. $P$ is compact, convex and satisfies the following property: for all $x \in P$, there exists $r>0$ such that for all $\tilde{x} \in P, \tilde{x} \neq x$,

$$
x+r \frac{\tilde{x}-x}{\|\tilde{x}-x\|} \in P ;
$$

3. $P$ is compact, convex and all its extreme points are isolated.

The last condition in property 2 says that the ball of radius $r$ intersected with the cone of all interior directions from $x$ is a subset of $P .{ }^{6}$

\footnotetext{
${ }^{6}$ I am thankful to an associate editor for this interpretation and for the present proof of Proposition 8 .
} 
Proof of Proposition 8. We will prove that 1 implies 2, 2 implies 3 and 3 implies 1. For convenience, let $E(C)$ denote the set of extreme points of a convex set $C$.

Property 2 implies 3 because if there were a sequence $\left\{x_{k}\right\}_{k=1}^{\infty}$ of extreme points of $P$ converging to $x$, its elements would eventually be in an $r / 2$-ball around $x$, but then its elements would not be extreme: defining $x^{\prime}=x+2\left(x_{k}-x\right)$, then $x_{k}=\left(x^{\prime}+x\right) / 2$, a contradiction since $x^{\prime}$ belongs to $P$. Indeed, $x+r\left(x_{k}-x\right) /\left\|x_{k}-x\right\| \in P$ by property 2 and $x^{\prime}=\theta\left(x+r\left(x_{k}-x\right) /\left\|x_{k}-x\right\|\right)+(1-\theta) x$ with $\theta=2\left\|x_{k}-x\right\| / r \in(0,1)$.

Property 3 implies 1 because $P$ is equal to the convex hull of its extreme points and $E(P)$ is finite. Indeed, $E(P)$ is closed (see Rockafellar (1970, Corollary 18.1.1)) and clearly bounded; so, if it were to be infinite, then it would have an accumulation point, a contradiction to property 3 .

In order to establish that property 1 implies 2, note that polyhedra (and so polytopes) are intersections of finitely many half-spaces. Let $P=\cap_{i \in F}\left\{z \in \mathbb{R}^{n}: c_{i} \cdot z \leq d_{i}\right\}$ for some finite set $F$, vectors $c_{i} \in \mathbb{R}^{n}$ and scalars $d_{i} \in \mathbb{R}$. Let $T=\left\{i \in F: c_{i} \cdot x=d_{i}\right\}$ and $T^{c}=F \backslash T$. Take $r$ to be the smallest distance of $x$ from the hyperplanes $H_{i}=\left\{z \in \mathbb{R}^{n}: c_{i} \cdot z=d_{i}\right\}$ for all $i \in T^{c}$, unless $T^{c}=\emptyset$, in which case we can take $r=1$. Let $\tilde{x} \in P$ and define $\bar{x}=x+r(\tilde{x}-x) /\|\tilde{x}-x\|$. We claim that $c_{i} \cdot \bar{x} \leq d_{i}$ holds for all $i \in F$, which clearly implies that $\bar{x} \in P$. For convenience, for all $i \in F$, let $S_{i}=\left\{z \in \mathbb{R}^{n}: c_{i} \cdot z \leq d_{i}\right\}$ and $d\left(x, H_{i}\right)=\inf _{z \in H_{i}}\|z-x\|$ denote the distance of $x$ from $H_{i}$. In the case $i \in T$, we have that $c_{i} \cdot x=d_{i}$ and $c_{i} \cdot \tilde{x} \leq d_{i}$ and so $c_{i} \cdot \bar{x}=d_{i}+r\left(c_{i} \cdot \tilde{x}-d_{i}\right) /\|\tilde{x}-x\| \leq d_{i}+0=d_{i}$. In the case $i \in T^{c}$, note that $\|\bar{x}-x\|=r \leq d\left(x, H_{i}\right)$, which implies that $\bar{x} \in S_{i}$. In fact, if $\bar{x} \notin S_{i}$, then define $\theta=\left(d_{i}-c_{i} \cdot x\right) /\left(c_{i} \cdot \bar{x}-c_{i} \cdot x\right) \in(0,1)$ (since $\left.0<d_{i}-c_{i} \cdot x<c_{i} \cdot \bar{x}-c_{i} \cdot x\right)$ and note that $\theta \bar{x}+(1-\theta) x \in H_{i}$. But then we obtain that $\|\theta \bar{x}+(1-\theta) x-x\|=\theta r<r \leq d\left(x, H_{i}\right)$, a contradiction.

The following lemma is a simple consequence of Proposition 8 and is key to the proof of Theorem 7 .

Lemma 9 Let $P$ be a polytope, $x \in P,\left\{x_{k}\right\}_{k=1}^{\infty}$ and $\left\{\alpha_{k}\right\}_{k=1}^{\infty}$ be such that $\lim _{k} x_{k}=x$, $0<\alpha_{k} \leq 1$ and $\alpha_{k} x_{k}+\left(1-\alpha_{k}\right) x \in P$ for all $k \in \mathbb{N}$. Then, there exist sequences 
$\left\{\hat{x}_{k}\right\}_{k=1}^{\infty}$ and $\left\{\theta_{k}\right\}_{k=1}^{\infty}$ such that $\lim _{k} \theta_{k}=0, \hat{x}_{k} \in P, \theta_{k} \in(0,1)$ and $x_{k}=\theta_{k} \hat{x}_{k}+(1-$ $\left.\theta_{k}\right) x$ for all $k$ sufficiently large. Furthermore, if $\left\{\alpha_{k}\right\}_{k=1}^{\infty}$ is bounded away from zero, then $\left\{\hat{x}_{k}\right\}_{k=1}^{\infty}$ and $\left\{\theta_{k}\right\}_{k=1}^{\infty}$ can be chosen so that $\lim _{k} \hat{x}_{k}=x$.

The following two special cases illustrate the usefulness of Lemma 9. The first case (to be considered in the proof of Theorem 7 below) is obtained by letting $\alpha_{k}=1$ for all $k$. Thus, in this case, we have a sequence $\left\{x_{k}\right\}_{k=1}^{\infty}$ contained in the polytope $P$, converging to a point $x$ also in $P$. Lemma 9 then asserts that, for all $k$ sufficiently large, each $x_{k}$ can be expressed as a convex combination between the limit point $x$ and some other point $\hat{x}_{k}$ in $P$.

A second special case of interest (to be considered in the proof of Proposition 12 below) occurs when $\alpha_{k}$ converges to zero. In this case, we have a sequence $\left\{x_{k}\right\}_{k=1}^{\infty}$ converging to a point $x$ in $P$ with the property that $\alpha_{k} x_{k}+\left(1-\alpha_{k}\right) x$ also belongs to $P$. Lemma 9 implies that $x_{k}$ must itself belong to $P$ for all $k$ sufficiently large: In fact, for all $k$ sufficiently large, each $x_{k}$ can be expressed as a convex combination between the limit point $x$ and some other point $\hat{x}_{k}$ in $P$.

Proof of Lemma 9. Note first that whenever $x_{k}=x$ we can let $\hat{x}_{k}=x$ and $\theta_{k}=1 / k$. Thus, we may assume that $x_{k} \neq x$ for all $k \in \mathbb{N}$.

Let $r>0$ be such that $x+r(\tilde{x}-x) /\|\tilde{x}-x\| \in P$ for all $\tilde{x} \in P, \tilde{x} \neq x$. For convenience, let $\bar{x}_{k}=\alpha_{k} x_{k}+\left(1-\alpha_{k}\right) x$ for all $k$. Note that there exists $K \in \mathbb{N}$ such that $\left\|x_{k}-x\right\|<r$ for all $k \geq K$. For all $k$, define $\gamma_{k}=\left\|\bar{x}_{k}-x\right\| / r$ and $\hat{x}_{k}=\left(1 / \gamma_{k}\right) \bar{x}_{k}+\left(1-1 / \gamma_{k}\right) x=x+r\left(\bar{x}_{k}-x\right) /\left\|\bar{x}_{k}-x\right\|$. Clearly, $\hat{x}_{k} \in P$ for all $k$. Then, $\bar{x}_{k}=\gamma_{k} \hat{x}_{k}+\left(1-\gamma_{k}\right) x$ and so $x_{k}=\left(\gamma_{k} / \alpha_{k}\right) \hat{x}_{k}+\left(1-\gamma_{k} / \alpha_{k}\right) x$ for all $k$.

Thus, define $\theta_{k}=\gamma_{k} / \alpha_{k}$ for all $k$, which immediately implies that $x_{k}=\theta_{k} \hat{x}_{k}+$ $\left(1-\theta_{k}\right) x$ for all $k$. Furthermore, $\theta_{k}=\left\|\bar{x}_{k}-x\right\| / r \alpha_{k}=\left\|x_{k}-x\right\| / r \in(0,1)$ for all $k \geq K$ and so $\lim _{k} \theta_{k}=0$.

Finally, consider the case where $\left\{\alpha_{k}\right\}_{k=1}^{\infty}$ is bounded away from zero. In this case, let $K \in \mathbb{N}$ be such that $\left\|\bar{x}_{k}-x\right\|^{1 / 2}<\min \{r, 1\}$ for all $k \geq K$. For all $k$, define $\gamma_{k}=\left\|\bar{x}_{k}-x\right\|^{1 / 2}$ and, as before, $\hat{x}_{k}=\left(1 / \gamma_{k}\right) \bar{x}_{k}+\left(1-1 / \gamma_{k}\right) x$ and $\theta_{k}=\gamma_{k} / \alpha_{k}$. Clearly, $\theta_{k}>0$ for all $k$ and $\lim _{k} \theta_{k}=0$, implying that $\theta_{k} \in(0,1)$ for sufficiently large $k$. 
Note that $\left\|\hat{x}_{k}-x\right\|=\left\|\bar{x}_{k}-x\right\| / \gamma_{k}=\left\|\bar{x}_{k}-x\right\|^{1 / 2}$ and so $\lim _{k} \hat{x}_{k}=x$. It remains to show that $\hat{x}_{k} \in P$ for all $k \geq K$. Since $\left\|\bar{x}_{k}-x\right\|^{1 / 2} \leq r$, then $1 /\left\|\bar{x}_{k}-x\right\|^{1 / 2} \leq$ $r /\left\|\bar{x}_{k}-x\right\|$, which implies that $\hat{x}_{k}$ can be expressed as a convex combination of $x$ and $x+r\left(\bar{x}_{k}-x\right) /\left\|\bar{x}_{k}-x\right\|$. Since the latter point belongs to $P$, then $\hat{x}_{k}$ also belongs to $P$.

Lemma 9, together with Ziad's original argument, allows us to prove Theorem 7.

Proof of Theorem 7. Let $i \in N$ and let $\beta_{i}: X_{-i} \rightrightarrows X_{i}$ be defined by $\beta_{i}\left(x_{-i}\right)=\left\{x_{i} \in X_{i}: u_{i}\left(x_{i}, x_{-i}\right)>V_{i}\left(x_{-i}\right)-\varepsilon\right\}$. Note that $B R_{i}^{\varepsilon}\left(x_{-i}\right)=\operatorname{cl}\left(\beta_{i}\left(x_{-i}\right)\right)$ for all $x_{-i} \in X_{-i}$. Furthermore, it follows by Aliprantis and Border (1999, Lemma 16.22, p. 535), that $B R_{i}^{\varepsilon}$ is lower hemi-continuous if and only if $\beta_{i}$ is lower hemi-continuous.

In order to establish that $\beta_{i}$ is lower hemi-continuous, let $F \subseteq X_{i}$ be closed, $x_{-i} \in X_{-i},\left\{x_{-i}^{k}\right\}_{k=1}^{\infty} \subseteq\left\{x_{-i}^{\prime} \in X_{-i}: \beta_{i}\left(x_{-i}^{\prime}\right) \subseteq F\right\}$ be such that $\lim _{k} x_{-i}^{k}=x_{-i}$ and $x_{i} \in \beta_{i}\left(x_{-i}\right)$. We will show that $x_{i} \in F$, which implies that $\beta_{i}\left(x_{-i}\right) \subseteq F$.

Let $0<\eta<\varepsilon$ be such that $u_{i}\left(x_{i}, x_{-i}\right)>V_{i}\left(x_{-i}\right)-\varepsilon+\eta$. Since $u_{i}$ is upper semi-continuous, so is $V_{i}$. Thus, let $\delta>0$ be such that $V_{i}\left(x_{-i}^{\prime}\right)<V_{i}\left(x_{-i}\right)+\eta / 2$ for all $x_{-i}^{\prime} \in X_{-i}$ satisfying $\left\|x_{-i}-x_{-i}^{\prime}\right\|<\delta$. By Lemma 9 (with $\alpha_{k}=1$ for all $k$ ), let $\left\{\hat{x}_{-i}^{k}\right\}_{k=1}^{\infty}$ and $\left\{\theta_{k}\right\}_{k=1}^{\infty}$ be such that $\lim _{k} \hat{x}_{-i}^{k}=x, \lim _{k} \alpha_{k}=0, \hat{x}_{-i}^{k} \in X_{i}, \theta_{k} \in(0,1)$ and $x_{-i}^{k}=\theta_{k} \hat{x}_{-i}^{k}+\left(1-\theta_{k}\right) x_{-i}$ for all $k$ sufficiently large. Taking a subsequence if necessary, we may assume that $\left\{V_{i}\left(\hat{x}_{-i}^{k}\right)\right\}_{k=1}^{\infty}$ converges to $\lim \sup _{k} V_{i}\left(\hat{x}_{-i}^{k}\right)$ and that $\hat{x}_{-i}^{k} \in X_{i}, \theta_{k} \in(0,1)$ and $x_{-i}^{k}=\theta_{k} \hat{x}_{-i}^{k}+\left(1-\theta_{k}\right) x_{-i}$ for all $k \in \mathbb{N}$.

Let $\hat{x}_{i}^{k} \in X_{i}$ be such that $u_{i}\left(\hat{x}_{i}^{k}, \hat{x}_{-i}^{k}\right)>V_{i}\left(\hat{x}_{-i}^{k}\right)-\varepsilon+\eta / 2$ and define $x_{i}^{k}=\theta_{k} \hat{x}_{i}^{k}+$ $\left(1-\theta_{k}\right) x_{i}$ for all $k$. Since $\lim _{k} \theta_{k}=0$, it follows that $\lim _{k} x_{i}^{k}=x_{i}$.

We claim that $x_{i}^{k} \in \beta_{i}\left(x_{-i}^{k}\right)$ for all $k$ sufficiently large. Suppose, in order to reach a contradiction, that there exists an infinite sequence $\left\{k_{j}\right\}_{j \in \mathbb{N}}$ such that $u_{i}\left(x_{i}^{k_{j}}, x_{-i}^{k_{j}}\right) \leq$ $V_{i}\left(x_{-i}^{k_{j}}\right)-\varepsilon$. Taking a further subsequence if needed, we may assume that there exists $l \in\left\{1, \ldots, L_{i}\right\}$ such that $\hat{x}_{-i}^{k_{j}} \in X_{-i}^{l}$ for all $j$. Since $X_{-i}^{l}$ is compact, then $x_{-i} \in X_{i}^{l}$. Thus, the quasi-concavity of $u_{i}$ in $X_{i} \times X_{-i}^{l}$ implies that

$$
\begin{aligned}
u_{i}\left(x_{i}^{k_{j}}, x_{-i}^{k_{j}}\right) & \geq \min \left\{u_{i}\left(x_{i}, x_{-i}\right), u_{i}\left(\hat{x}_{i}^{k_{j}}, \hat{x}_{-i}^{k_{j}}\right)\right\} \\
& >\min \left\{V_{i}\left(x_{-i}\right)-\varepsilon+\eta, V_{i}\left(\hat{x}_{-i}^{k_{j}}\right)-\varepsilon+\eta / 2\right\}=V_{i}\left(\hat{x}_{-i}^{k_{j}}\right)-\varepsilon+\eta / 2,
\end{aligned}
$$


for all $j$ such that $\left\|\hat{x}_{-i}^{k_{j}}-x_{-i}\right\|<\delta$ (since in this case, $\left.V_{i}\left(\hat{x}_{-i}^{k_{j}}\right)<V_{i}\left(x_{-i}\right)+\eta / 2\right)$. Hence, $V_{i}\left(\hat{x}_{-i}^{k_{j}}\right)+\eta / 2<V_{i}\left(x_{-i}^{k_{j}}\right)$ for all $j$ sufficiently large, and so

$$
\underset{k}{\limsup } V_{i}\left(\hat{x}_{-i}^{k}\right)+\frac{\eta}{2}=\lim _{j} V_{i}\left(\hat{x}_{-i}^{k_{j}}\right)+\frac{\eta}{2} \leq \limsup _{j} V_{i}\left(x_{-i}^{k_{j}}\right) \leq \underset{k}{\limsup } V_{i}\left(x_{-i}^{k}\right),
$$

contradicting the $i$-upper semi-continuity of $u_{i}$. This contradiction establishes that $x_{i}^{k} \in \beta_{i}\left(x_{-i}^{k}\right)$ for all $k$ sufficiently large. Since $\beta_{i}\left(x_{-i}^{k}\right) \subseteq F$ for all $k$ and $F$ is closed, then $x_{i}=\lim _{k} x_{i}^{k} \in F$, as desired. Thus, $\beta_{i}$, and so $B R_{i}^{\varepsilon}$, is lower hemi-continuous.

It is clear that $B R_{i}^{\varepsilon}\left(x_{-i}\right)$ is nonempty, closed and convex. Therefore, $B R_{\varepsilon}(x)=$ $B R_{1}^{\varepsilon}\left(x_{-1}\right) \times \cdots \times B R_{n}^{\varepsilon}\left(x_{-n}\right)$ is also nonempty, closed and convex. Furthermore, $B R_{\varepsilon}: X \rightrightarrows X$ is lower hemi-continuous. Thus, by Michael's selection theorem, there exists a continuous selection $f$ of $B R_{\varepsilon}$. Hence, by Brouwer's fixed point theorem, $f$ has a fixed point $x$ and $x$ is an $\varepsilon$-equilibrium of $G$.

We note that the only difference between the statement of Theorem 7 and the original statement in Ziad (1997) is that players' action spaces are now required to be polytopes (and not just a convex and compact subset of a euclidean space). As the following example shows, requiring the action spaces to be convex and compact is not sufficient to guarantee the lower hemi-continuity of the $\varepsilon$-best reply correspondence.

Example 10 Let $G=\left(X_{1}, X_{2}, u_{1}, u_{2}\right)$ be the following 2-player game. Let

$$
X_{1}=\left\{(x, y) \in \mathbb{R}^{2}: 0 \leq x \leq 1 \text { and }(1-x)^{2} \leq y \leq 1-x\right\}
$$

and $X_{2}=[0,1]$. We denote $x_{1}=(x, y)$ and $x_{2}=z$.

Let player 1's payoff function $u_{1}$ be defined by $u_{1}(x, y, z)=0$ for all $(x, y, z) \in X_{1} \times$ $X_{2}$. We define player 2's payoff function as follows. Define $A=X_{1} \times\{0\} \subseteq X_{1} \times X_{2}$, $B=\{(1,0,1)\} \subseteq X_{1} \times X_{2}$ and $C=\operatorname{co}(A \cup B)$. The payoff function for player 2 is

$$
u_{2}(x, y, z)= \begin{cases}2 & \text { if }(x, y, z) \in B \\ 1 & \text { if }(x, y, z) \in C \backslash B \\ 0 & \text { otherwise. }\end{cases}
$$

Note that $X_{1}=\left\{(x, y) \in \mathbb{R}^{2}: 0 \leq x \leq 1\right.$ and $\left.f(x) \leq y \leq 1-x\right\}$, where $f: \mathbb{R} \rightarrow \mathbb{R}$ is defined by $f(x)=(1-x)^{2}$. Since $f$ is strictly convex and continuous, $X_{1}$ is convex 
and compact. Clearly, $X_{2}$ is also compact and convex. Note that $u_{2}$ is quasi-concave on $X_{1} \times X_{2}$. Furthermore, $A \cup B$, hence $\operatorname{co}(A \cup B)$, is compact, and so $u_{2}$ is upper semi-continuous. Clearly, $u_{1}$ is both upper semi-continuous and quasi-concave.

We claim that $u_{i}$ is also $i$-upper semi-continuous for all $i$. It is clear that $V_{1}(z)=0$ for all $z \in X_{2}$ and $V_{2}(x, y)=1$ for all $(x, y) \in X_{1}$, except when $(x, y)=(1,0)$, in which case $V_{2}$ equals 2 . Condition (1) is trivially satisfied for player 1 and one can easily show that $V_{2}$ also satisfies it. To see this, let $(x, y) \in X_{1},\left\{\left(x_{k}, y_{k}\right)\right\}_{k=1}^{\infty}$ converging to $(x, y)$ and $\left\{\alpha_{k}\right\}_{k=1}^{\infty} \subseteq(0,1]$ converging to zero. If $(x, y) \neq(1,0)$, we readily obtain that $\lim \sup _{k} V_{2}\left(\alpha_{k}\left(x_{k}, y_{k}\right)+\left(1-\alpha_{k}\right)(x, y)\right)=\lim \sup _{k} V_{2}\left(x_{k}, y_{k}\right)=1$. If $(x, y)=(1,0)$, the fact that $\limsup _{k} V_{2}\left(x_{k}, y_{k}\right) \geq 1$ implies that it is enough to consider the case when $\lim \sup _{k} V_{2}\left(\alpha_{k}\left(x_{k}, y_{k}\right)+\left(1-\alpha_{k}\right)(x, y)\right)=2$. However, this condition holds if and only if there exists a subsequence $\left\{\left(x_{k_{j}}, y_{k_{j}}\right)\right\}_{j=1}^{\infty}$ such that $\left(x_{k_{j}}, y_{k_{j}}\right)=(1,0)$. But, clearly, in this case we also have $\lim \sup _{k} V_{2}\left(x_{k}, y_{k}\right)=2$. Thus, it follows that $V_{2}$ satisfies condition (1) and so $u_{2}$ is $i$-upper semi-continuous.

Let $0<\varepsilon<1$. Then, $B R_{1}^{\varepsilon}(z)=X_{1}$ for all $z \in X_{2}$ and

$$
B R_{2}^{\varepsilon}(x, y)= \begin{cases}\{1\} & \text { if }(x, y)=(1,0), \\ \{z \in[0,1]:(x, y, z) \in C\} & \text { otherwise }\end{cases}
$$

for all $(x, y) \in X_{1}$. Clearly, if $g: X_{1} \rightarrow X_{2}$ is a selection from $B R_{2}^{\varepsilon}$, then $g(x,(1-$ $\left.x)^{2}\right)=0$ for all $x \in[0,1)$, while $g(1,0)=1$. Hence, it follows that $B R_{2}^{\varepsilon}$ has no continuous selection. Since $B R_{2}^{\varepsilon}(x, y)$ is nonempty, convex and compact for all $(x, y) \in$ $X_{1}$, it follows by Michael's selection theorem that $B R_{2}^{\varepsilon}$ is not lower hemi-continuous.

Before we conclude this section, we note that the above example can be easily modified to show that, even when action spaces are polytopes, the $\varepsilon$-best reply correspondence may fail to be lower hemi-continuous if players' payoff functions are not $i$-upper semi-continuous:

Example 11 Let $G=\left(X_{1}, X_{2}, u_{1}, u_{2}\right)$ be as in Example 10 except that $X_{1}=\{(x, y) \in$ $[0,1] \times[0,1]: y \leq 1-x\}$ and both $u_{1}$ and $u_{2}$ are extended by defining $u_{1}(x, y, z)=$ $u_{2}(x, y, z)=0$ for all $(x, y, z) \in X_{1} \times X_{2}$ such that $y<(1-x)^{2}$.

Clearly, both payoff functions are quasi-concave and upper semi-continuous. The same argument used above shows that $B R_{2}^{\varepsilon}$ is neither lower hemi-continuous nor 
does it have a continuous selection for all $0<\varepsilon<1$. Finally, note that $u_{2}$ is not $i$-upper semi-continuous. Letting $(x, y)=(1,0),\left(x_{k}, y_{k}\right)=\left(1-1 / k, 1 / 2 k^{2}\right)$ and $\alpha_{k}=1 / 2 k$ for all $k \in \mathbb{N}$, we obtain that $y_{k}<f\left(x_{k}\right)$ (recall that $f(x)=(1-x)^{2}$ ), $\alpha_{k} y_{k}+\left(1-\alpha_{k}\right) 0=1 / 4 k^{3} \geq f\left(1-1 / 2 k^{2}\right)=f\left(\alpha_{k} x_{k}+\left(1-\alpha_{k}\right) 1\right)$ for all $k$ and so $\lim \sup _{k} V_{i}\left(\alpha_{k}\left(x_{k}, y_{k}\right)+\left(1-\alpha_{k}\right)(1,0)\right)=1>0=\lim \sup _{k} V_{i}\left(x_{k}, y_{k}\right)$.

\section{Relationship between Radzik's and Ziad's The- orems}

As we have shown in the previous section, the polyhedral convexity of the action spaces is essential to the lower hemi-continuity of the $\varepsilon$-best reply correspondence and, therefore, to any approach to the existence of $\varepsilon$-equilibria based on that property. The importance of polyhedral convexity is strengthened here by showing that piecewise polyhedral concavity of the players' value functions implies $i$-upper semi-continuity. This result is then used to show that Radzik's Theorem is a corollary of the version of Ziad's theorem presented in Theorem 7 .

Proposition 12 If $V_{i}$ is piecewise polyhedral quasi-concave, then $u_{i}$ is $i$-upper semicontinuous.

Proof. Let $x_{-i} \in X_{-i},\left\{x_{-i}^{k}\right\}_{k=1}^{\infty} \subseteq X_{-i}$ be a sequence converging to $x_{-i}$ and $\left\{\alpha_{k}\right\}_{k=1}^{\infty} \subseteq(0,1]$ converging to zero. Let $\bar{x}_{-i}^{k}=\alpha_{k} x_{-i}^{k}+\left(1-\alpha_{k}\right) x_{-i}$ for all $k \in \mathbb{N}$.

Let $\gamma=\lim \sup _{k} V_{i}\left(\bar{x}_{-i}^{k}\right)$. Then, there exists a subsequence $\left\{\bar{x}_{-i}^{k_{j}}\right\}_{j}$ of $\left\{\bar{x}_{-i}^{k}\right\}_{k}$ such that $\lim _{j \rightarrow \infty} V_{i}\left(\bar{x}_{-i}^{k_{j}}\right)=\gamma$. We may assume that $x_{-i}^{k_{j}} \neq x_{-i}$ for infinitely many indexes $j$, since otherwise $x_{-i}^{k_{j}}=\bar{x}_{-i}^{k_{j}}=x_{-i}$ for all $j$ sufficiently large and so $\lim \sup _{k} V_{i}\left(x_{-i}^{k}\right) \geq$ $\lim \sup _{j} V_{i}\left(x_{-i}^{k_{j}}\right)=V_{i}\left(x_{-i}\right)=\lim \sup _{k} V_{i}\left(\bar{x}_{-i}^{k}\right)$. Hence, taking a subsequence if necessary, we may assume that $x_{-i}^{k_{j}} \neq x_{-i}$ for all $j \in \mathbb{N}$; clearly, this implies that $\bar{x}_{-i}^{k_{j}} \neq x_{-i}$ for all $j \in \mathbb{N}$. Let $\varepsilon>0$ and let $J_{1} \in \mathbb{N}$ be such that $V_{i}\left(\bar{x}_{-i}^{k_{j}}\right)>\gamma-\varepsilon$ for all $j \geq J_{1}$.

Let $\left\{X_{-i}^{l}\right\}_{l=1}^{L_{i}}$ be a compact, convex cover of $X_{-i}$ such that $V_{i}$ is polyhedral quasiconcave on $X_{-i}^{l}$ for all $l=1, \ldots, L_{i}$. Since the cover is finite, we may assume that there exists $l \in\left\{1, \ldots, L_{i}\right\}$ such that $\bar{x}_{-i}^{k_{j}} \in X_{-i}^{l}$ for all $j \in \mathbb{N}$. Letting $P=\{y \in$ 
$\left.X_{-i}^{l}: V_{i}(y) \geq \gamma-\varepsilon\right\}$, then $P$ is a polytope and $\bar{x}_{-i}^{k_{j}} \in P$ for all $j \geq J_{1}$. Furthermore, $x_{-i} \in P$ since $P$ is compact.

By Lemma 9, there exist $J>J_{1}$ and sequences $\left\{\hat{x}_{-i}^{k_{j}}\right\}_{k=1}^{\infty}$ and $\left\{\theta_{k_{j}}\right\}_{k=1}^{\infty}$ such that $\hat{x}_{-i}^{k_{j}} \in P, \theta_{k_{j}} \in(0,1)$ and $x_{-i}^{k_{j}}=\theta_{k_{j}} \hat{x}_{-i}^{k_{j}}+\left(1-\theta_{k_{j}}\right) x_{-i}$ for all $j \geq J$. Hence, for all $j \geq J$, it follows that $x_{-i}^{k_{j}} \in P$, which implies that $V_{i}\left(x_{-i}^{k_{j}}\right) \geq \gamma-\varepsilon$ and $\operatorname{so~} \lim \sup _{k} V_{i}\left(x_{-i}^{k}\right) \geq$ $\gamma-\varepsilon$. Since $\varepsilon>0$ is arbitrary, it follows that $\lim \sup _{k} V_{i}\left(x_{-i}^{k}\right) \geq \gamma=\lim \sup _{k} V_{i}\left(\bar{x}_{-i}^{k}\right)$. Thus, $u_{i}$ is $i$-upper semi-continuous.

We note that for two-player games on a square with piecewise quasi-concave payoff functions, each player has a piecewise polyhedral quasi-concave value function and so an $i$-upper semi-continuous payoff function. Indeed, since, for all $i=1,2$, both $X_{i}$ and $X_{-i}$ are contained in $\mathbb{R}$, every convex, compact subset of $X_{i}$ or $X_{-i}$ is a polytope. In particular, $X_{i}$ and $X_{-i}$ are polytopes, if $\left\{X_{-i}^{l}\right\}_{l=1}^{L_{i}}$ is a compact, convex cover of $X_{-i}$, then $X_{-i}^{l}$ is a polytope and so are the upper sets $\left\{x_{-i} \in X_{-i}^{l}: V_{i}\left(x_{-i}\right) \geq a\right\}$, $a \in \mathbb{R}$, of $V_{i}$ in $X_{-i}^{l}$. Thus, combining this observation with Ziad's theorem, we obtain the main result in Radzik (1991).

Corollary 13 (Radzik) If $G$ is a two-player game such that, for all $i=1,2$, (1) $X_{i}$ is a compact interval on the real line and (2) $u_{i}$ is upper semi-continuous and piecewise quasi-concave, then $G$ has an $\varepsilon$-equilibrium for all $\varepsilon>0$.

We can also use Proposition 12 to obtain existence results for $n$-person games played in polytopes that parallel the statement of Radzik's Theorem and are, again, a corollary of Ziad's. One such result is Proposition 6, stated in the introduction and reproduced in Corollary 14 below for convenience. Its statement parallels that of Radzik in that action spaces are polytopes and payoff functions are upper semicontinuous and piecewise quasi-concave, but it allows for more than two players and for action spaces that are subsets of any euclidean space. Since in this setting players' value functions are not necessarily piecewise polyhedral quasi-concave, this requirement must be made explicitly. A second existence result is then obtained by noting that if players' payoff functions are piecewise polyhedral quasi-concave, so are their value functions (player $i$ 's payoff function is piecewise polyhedral quasi-concave if there 
is a finite cover of $X_{-i}$ by compact convex subsets $X_{-i}^{l}$ such that $u_{i}$ is polyhedral quasi-concave on $X_{i} \times X_{-i}^{l}$ for all $l$ ).

Corollary 14 If $G$ is such that, for all $i \in N$, (1) $X_{i}$ is a polytope, (2) $u_{i}$ is upper semi-continuous and piecewise quasi-concave and (3) $V_{i}$ is polyhedral piecewise quasiconcave, then $G$ has an $\varepsilon$-equilibrium for all $\varepsilon>0$.

In particular, if $G$ is such that, for all $i \in N$, (1) $X_{i}$ is a polytope and (2) $u_{i}$ is upper semi-continuous and piecewise polyhedral quasi-concave, then $G$ has an $\varepsilon$ equilibrium for all $\varepsilon>0$.

\section{Concluding Remarks}

The approach for the existence results discussed in this note relies on the lower hemicontinuity of the $\varepsilon$-best reply correspondence. As the example in Section 3 shows, this condition may fail even if the game satisfies the assumptions in Ziad (1997). Nevertheless, we have shown that this problem can be solved by assuming that players' action spaces are polytopes. Furthermore, we show that if an upper semi-continuous game is such that the upper sets of players' payoff functions are polytopes (or at least it satisfies a generalization of this condition), then it has an $\varepsilon$-equilibrium for all $\varepsilon>0$.

The drawback of this note is that it does not answer the question of whether or not Ziad's theorem holds as it was stated originally. For instance, it is clear that the game presented in Section 2 has Nash equilibria. Moreover, so does the game presented at the end of Section 2 and obtained by modifying the original example so that players' payoff functions are not $i$-upper semi-continuous. In fact, one can conjecture that every game with nonempty, compact, convex and finite-dimensional action spaces and with upper semi-continuous and piecewise quasi-concave payoff functions have $\varepsilon$-equilibria for all $\varepsilon>0$.

Although no answer to the above conjecture will be offered, we note that the problem can be simplified by reducing it to games with finite-valued payoff functions. In fact, if $G$ is a game satisfying the above properties, we can first normalize payoffs 
so that $u_{i}(X) \subseteq(0,1)$ for all $i \in N$ and then define, for all $k \in \mathbb{N}, u_{i}^{k}(x)=(j-1) / 2^{k}$ if $(j-1) / 2^{k} \leq u_{i}(x)<j / 2^{k}$, for all $j=0, \ldots, 2^{k}$ (so, $u_{i}^{k}(x)$ is defined by rounding down $u_{i}(x)$ to the nearest multiple of $\left.2^{-k}\right)$. Then, it is easy to see that for all $i \in N$ and $k \in$ $\mathbb{N}, u_{i}^{k}$ is finite-valued, upper semi-continuous and piecewise quasi-concave and $\left\{u_{i}^{k}\right\}_{k=1}^{\infty}$ converges uniformly to $u_{i}$. In fact, if $a \in(0,1)$ and $j=\min \left\{j^{\prime}:\left(j^{\prime}-1\right) / 2^{k} \geq a\right\}$, then $\left\{x \in X_{i} \times Y_{-i}: u_{i}^{k}(x) \geq a\right\}=\left\{x \in X_{i} \times Y_{-i}: u_{i}(x) \geq(j-1) / 2^{k}\right\}$ is closed if $Y_{-i}=X_{-i}$ and convex if $Y_{-i}=X_{-i}^{l}$ (where $\left\{X_{-i}^{l}\right\}_{l}$ is a compact convex cover of $X_{-i}$ given by the piecewise quasi-concavity of $u_{i}$ ). Thus, $u_{i}^{k}$ is upper semi-continuous and piecewise quasi-concave. Furthermore, one has that $\sup _{x \in X}\left|u_{i}^{k}(x)-u_{i}(x)\right| \leq 2^{-k}$ implying that $\left\{u_{i}^{k}\right\}_{k=1}^{\infty}$ converges uniformly to $u_{i}$. We finally claim that if one establishes that every game with finite-valued, upper semi-continuous and piecewise quasi-concave payoff functions (whose action spaces also satisfy the same properties as those of $G$ ) have a Nash equilibrium, then the properties of $\left\{u_{i}^{k}\right\}_{k=1}^{\infty}, i \in N$, imply that $G$ has an $\varepsilon$-equilibrium for all $\varepsilon>0$. Indeed, if $k \in \mathbb{N}$ is such that $\sup _{x \in X}\left|u_{i}^{k}(x)-u_{i}(x)\right|<\varepsilon / 2$ for all $i \in N$, and $x^{*}$ is a Nash equilibrium of $G_{k}=\left(X_{i}, u_{i}^{k}\right)_{i \in N}$, then, for all $i \in N$ and $x_{i} \in X_{i}, u_{i}\left(x^{*}\right)>u_{i}^{k}\left(x^{*}\right)-\varepsilon / 2 \geq u_{i}^{k}\left(x_{i}, x_{-i}^{*}\right)-\varepsilon / 2>u_{i}\left(x_{i}, x_{-i}^{*}\right)-\varepsilon$ and so $x^{*}$ is an $\varepsilon$-equilibrium of $G$.

\section{References}

Aliprantis, C., And K. Border (1999): Infinite Dimensional Analysis. Springer, Berlin.

Dasgupta, P., And E. Maskin (1986): "The Existence of Equilibrium in Discontinuous Economic Games, I: Theory," Review of Economics Studies, 53, 1-26.

Gale, D., V. Klee, and R. Rockafellar (1968): "Convex Functions on Convex Polytopes," Proceedings of the American Mathematical Society, 19, 867-873.

NAsh, J. (1950): "Equilibrium Points in N-person Games," Proceedings of the National Academy of Sciences, 36, 48-49. 
RAdzIK, T. (1991): "Pure-Strategy $\varepsilon$-Nash Equilibrium in Two-Person Non-zeroSum Games," Games and Economic Behavior, 3, 356-367.

Reny, P. (1999): "On the Existence of Pure and Mixed Strategy Equilibria in Discontinuous Games," Econometrica, 67, 1029-1056.

Rockafellar, R. T. (1970): Convex Analysis. Princeton University Press, Princeton.

ZIAD, A. (1997): "Pure-Strategy $\varepsilon$-Nash Equilibrium in $n$-Person Nonzero-Sum Discontinuous Games," Games and Economic Behavior, 20, 238-249. 